\title{
Assessment of chemosensory function using electroencephalographic techniques*
}

\author{
Ph. Rombaux ${ }^{1,2}$, C. Huart ${ }^{1,2}$, A. Mouraux ${ }^{2}$ \\ Rhinology 50: 13-21, 2012 \\ DOI: 10.4193/Rhino11.126 \\ Department of Otorhinolaryngology, Cliniques universitaires Saint-Luc, Brussels, Belgium \\ Institute of Neuroscience, Université Catholique de Louvain, Brussels, Belgium \\ *Received for publication: \\ June 17, 2011 \\ accepted: November 4, 2011
}

\section{Summary}

Electroencephalographic techniques are widely used to provide an objective evaluation of the chemosensory function and to explore neural mechanisms related to the processing of chemosensory events. The most popular technique to evaluate brain responses to chemosensory stimuli is across trial time-domain averaging to reveal chemosensory event-related potentials (CSERP) embedded within the ongoing EEG. Nevertheless, this technique has a poor signal-to-noise ratio and cancels out stimulus-induced changes in the EEG signal that are not strictly phased-locked to stimulus onset. The fact that consistent CSERP are not systematically identifiable in healthy subjects currently constitutes a major limitation to the use of this technique for the diagnosis of chemosensory dysfunction.

In this review, we will review the different techniques related to the recording and identification of CSERP, discuss some of their limitations, and propose some novel signal processing methods which could be used to enhance the signal-to-noise ratio of chemosensory event-related brain responses.

Key words: chemosensory event-related potentials, electroencephalogram, olfaction, trigeminal

\section{Introduction}

Unlike other sensory modalities (auditory, visual, somatosensory), the use of electrophysiological techniques to explore the olfactory system in humans remains sparse. This is mostly due to the difficulty of producing selective and controlled olfactory stimuli ${ }^{(1)}$; and the fact that pioneering studies have yielded sometimes conflicting results ${ }^{(2-4)}$. In 1978, Kobal and Plattig ${ }^{(5)}$ introduced an olfactory stimulator, which allows brief chemosensory stimulation of the olfactory neuroepithelium of the nasal mucosa, without concurrent mechanical and/ or thermal stimulation. Several studies have now shown that this technique can be used to explore the cortical processing of olfactory and trigeminal chemosensory input in humans, through the recording of chemosensory event-related brain potentials (CSERPs) ${ }^{(6-8)}$. Investigating the test-retest reliability of CSERP, studies have shown that CSERP had good test-retest reliability ${ }^{(9,10)}$. CSERP were thus considered to be valuable for the objective clinical evaluation of patients ${ }^{(9,10)}$. However, due to the poor signal-to-noise ratio of the elicited responses (11-13), CSERPs are often difficult to assess, especially olfactory CSERPs, even in normosmic patients. For example, Lötsch and Hummel (12) showed that approximately $30 \%$ of normosmic people have no identifiable olfactory CSERP. Hence, at present, the clinical usefulness of CSERP remains questionable.

Some hypotheses have been proposed to explain the absence of identifiable olfactory CSERP among normosmic people. First, some of their normosmic subjects can have CSERP only to one odorant. Secondly, some patients may have a very high number of artifacts in the recording and; finally in a small number of subjects no olfactory CSERP could be elicited ${ }^{(12)}$.

This review will focus on the techniques related to the recording of CSERPs, and will propose several novel signal proces- 
sing methods which could be used to enhance the signal-tonoise ratio of CSERPs.

\section{Chemosensory stimulation device}

The vast majority of odorants stimulate both the olfactory and trigeminal system. The selective evaluation of the olfactory or trigeminal system thus requires specific molecules to activate either of the two systems. To activate olfactory chemosensory afferents selectively, the most used substances are 2-phenylethanol (PEA) and hydrogen sulfide $\left(\mathrm{H}_{2} \mathrm{~S}\right)^{(12-14)}$. To activate trigeminal chemosensory afferents selectively, gaseous $\mathrm{CO}_{2}$ is the most commonly used ${ }^{(12-15)}$.

The ideal chemosensory stimulator to record time-locked responses such as event-related potentials (ERPs) should meet the following criteria: (a) the stimulator should be able to activate trigeminal or olfactory chemosensory receptors selectively, (b) the stimulator should be able to deliver chemosensory stimuli in a controlled fashion, (c) use of the stimulator should be safe, and (d) the stimulator should be able to generate a very phasic stimulus, compatible with the recording of timelocked responses.

Devising a chemosensory stimulus that is able to activate olfactory or trigeminal chemosensitive receptors of the nasal mucosa without concurrent mechanical or thermal stimulation of mechanical or heat sensitive trigeminal afferents is technically challenging. Furthermore, producing a transient chemosensory stimulus is difficult, as it requires the ability to generate a very steep gradient in odorant concentration.

At present, we can distinguish two types of olfactometers: blast olfactometers and constant flow olfactometers (Figure 1A). Blast olfactometers deliver pulses of odorized air in the subject's nose. This approach has the advantage of being able to deliver very transient stimuli, but also has one big disadvantage: the sudden pulse of air necessarily also activates mechanoreceptors of the nasal cavity. Therefore, if the technique is used to record olfactory CSERPs, these will be difficult to disentangle from the ERPs related to the mechanical activation of trigeminal somatosensory afferents. For this reason, constant flow olfactometers are now preferred for the recording of CSERPs. The technique was introduced by Kobal and Plattig in $1978^{(5)}$ and consists in delivering a pulsed odour embedded within a constant airflow. The rapid switching between the odour and the control airflow relies on a vacuum line. Importantly, the airflow is maintained at a constant temperature and humidity to avoid stimulation of trigeminal mechanical or heat sensitive receptors (Figure 1B). Furthermore, using very rapid switching valves, it is possible to deliver very phasic stimuli,

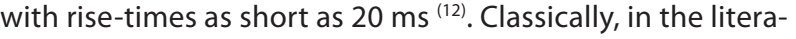
ture, subjects receive a constant intranasal airflow of $6-81 / \mathrm{min}$, which is at $80 \%$ relative humidity and warmed to $36^{\circ} \mathrm{C}$; with a rise time of $20 \mathrm{~ms}$ and a stimulus duration of $200 \mathrm{~ms}^{(12,13,15)}$.

The use of a constant flow olfactometer to record CSERPs remains difficult. Because of the low signal-to-noise ratio of the elicited responses, all measures should be taken to optimize stimulus presentation. To maximize the gradient between the absence of odorant in the control air and the presence of odorant in the pulsed odour, the air of the recording room should, itself, be devoid of any odorant, a condition that is often difficult to obtain in practice, but crucial to maximize the obtained CSERPs. In addition, the odorant is conveyed through a long Teflon tube, running from the olfactometer to the nostril. This Teflon tube may also be a source of contamination of the control air, as odours may impregnate it.

\section{Electroencephalographic responses to chemosensory stimulation}

The evaluation of olfactory function is most often performed using psychophysical testing such as the Sniffin' Sticks test $(16,17)$ or the University of Pennsylvania Smell Identification Test (UPSIT) ${ }^{(18)}$. These tests have the advantage of being easy to implement, and of having been validated in multicenter studies (16). However, they have the disadvantage of being only semiobjective and of being subject to patient response biases. This constitutes a major issue when evaluating patients with olfactory disorder, particularly if the evaluation is performed in a medico legal context. It is usually considered that this bias could be largely avoided by the recording of CSERPs.

The recording of ERPs following stimulation of sensory afferents (e.g. visual evoked potentials elicited by an alternating checkerboard) is a technique that is widely used for the functional assessment of sensory systems in clinical diagnostic medicine. Indeed, brief sensory stimuli elicit transient changes in the ongoing electroencephalographic activity. However, it is important to keep in mind that only a fraction of the cortical activity that is triggered by the stimulus will lead to a measurable electroencephalographic response ${ }^{(19)}$. To generate such a response, the elicited neuronal activity must satisfy a number of conditions ${ }^{(20)}$. First, the neuronal activity must involve a large population of neurons; and these neurons must be activated synchronously, such that the electrical fields generated by each neuron summate into a strong electrical field. Importantly, the activated neuronal population must constitute an open field structure. Indeed, if the geometric configuration of the activated neurons constitutes a closed field structure (e.g. a subcortical nucleus), the electrical fields produced by each neuron of the structure will cancel each other and the net electrical field will be null ${ }^{(21)}$. The geometric configuration of the olfactory cortex and, in particular, the entorhinal cortex, the piriform cortex and the orbitofrontal cortex indicate that 
these should constitute an open field structure. In contrast, the spherical geometric configuration of the amygdalar nucleus

suggests that it could act as a closed field structure and, hence, that activity generated within this structure is likely to not translate into a measurable electroencephalographic signal. Third, the distance between the scalp electrodes and the activated neural population should be small, as the magnitude of the generated electrical field decreases with the square of the distance. This could constitute an issue for the recording of CSERPs, as most structures thought to be involved in the processing of olfactory input are relatively deeply located, such as the piriform cortex, the entorhinal cortex, the amygdala and the orbito-frontal cortex.

When these criterions are met, the synchronous activation of a population of neurons by a transient sensory, motor or cognitive event can thus generate a measurable deflection in the human electroencephalogram (EEG), i.e., an ERP. These deflections are thought to mainly reflect summed postsynaptic activity occurring in similarly oriented cortical pyramidal neurons ${ }^{(21)}$.

It is also well-known that brief sensory, motor or cognitive events can modulate transiently the magnitude of the spontaneous oscillatory activity of the human electroencephalogram, within different frequency bands. These stimulus-induced modulations may appear as either a transient increase (eventrelated synchronization, ERS) or a transient decrease (event-related desynchronization, ERD) of the power of EEG oscillations $(22,23)$. The functional significance of ERS and ERD is thought to differ according to the affected frequency band. ERS and ERD in the alpha band $(8-12 \mathrm{~Hz})$ are thought to reflect respectively cortical deactivation and activation ${ }^{(23)}$. ERS in the gamma band has been hypothesized to constitute a mean of forming and synchronizing transient functional neuronal assemblies, and to play an important role in conscious perception ${ }^{(24,25)}$.

\section{Characterizing chemosensory ERPs in the time domain}

Brief chemosensory stimuli can thus elicit transient deflections within the ongoing EEG, referred to as CSERPs. Such as for other ERPs, the magnitude of these deflections is much smaller than the magnitude of the background ongoing EEG. To increase the signal-to-noise ratio of the elicited responses and, thereby, allow their identification, the event is repeated several times. The EEG signal is then segmented into separate epochs relative to the onset of each stimulus, and the obtained epochs are averaged into a single average waveform. This averaging procedure relies on the assumption that, across the different epochs, ERPs should be stationary relative to stimulus onset and, hence, they should be unaffected by the averaging procedure. In contrast, all EEG signals that are unrelated to the stimulus should be non-stationary relative to stimulus onset and, therefore, should be cancelled out by the averaging procedure. In this way, time-domain averaging increases the signal-to-noise ratio of ERPs.

The bulk of CSERPs consists of a negative component occurring between 320 and 450 ms after stimulus onset (often referred to as $\mathrm{N} 1$ ) $^{(26-29)}$, followed by a positive component occurring between 530 and 800 ms after stimulus onset (often referred to as P2). Both components are maximal at the scalp vertex. The positive component is sometimes described as comprising two distinct components, referred to as $\mathrm{P} 2$ and $\mathrm{P} 3$ (30). Preceding this N1 - P2 complex, an initial positive peak (P1) has occasionally been reported, peaking between 250 and 320 ms after stimulus onset ${ }^{\left({ }^{(3-33)}\right.}$ (Figure 2).

Conventional time-domain averaging presents several drawbacks, which could contribute to explain the low signal-tonoise ratio of CSERPs.

First, the across-trial latencies of CSERPs may be affected by a significant amount of temporal jitter. This jitter would result from the variability in timing of the different steps separating the occurrence of the sensory event (i.e. the onset of the chemosensory stimulus) and the occurrence of a cortical response (i.e. the ERP). If this jitter is significant, the elicited ERPs will be cancelled out by the time-domain averaging procedure, as the responses are no longer stationary across trials. Chemosensory stimuli are usually delivered using a Teflon tube pointing towards the olfactory cleft. By involuntarily displacing the position of the tube relative to the olfactory cleft, small head movements may increase the jitter. Furthermore, the process of transducting the chemosensory stimulus into a neural impulse may also be an important source of jitter. Therefore, it is reasonable to expect that the responses to chemosensory stimulation are subject to a significant amount of jitter, and that this jitter leads to an important distortion of the averaged ERP waveforms, possibly to the point of rendering the responses undetectable.

Second, time-domain averaging is unable to reveal any transient event-related modulation of the power of ongoing EEG oscillations (i.e. ERD and ERS), as these oscillations are cancelled-out by conventional time-domain averaging procedures. Taken together, time-domain averaging is thus blind to a significant fraction of the elicited EEG response (ERPs subject to a significant amount of temporal jitter, ERD and ERS). This could contribute to explain why CSERPs are often difficult to identify in healthy subjects.

\section{Characterizing chemosensory EEG responses in the time-frequency domain}

Different methods have been proposed to identify, characte- 


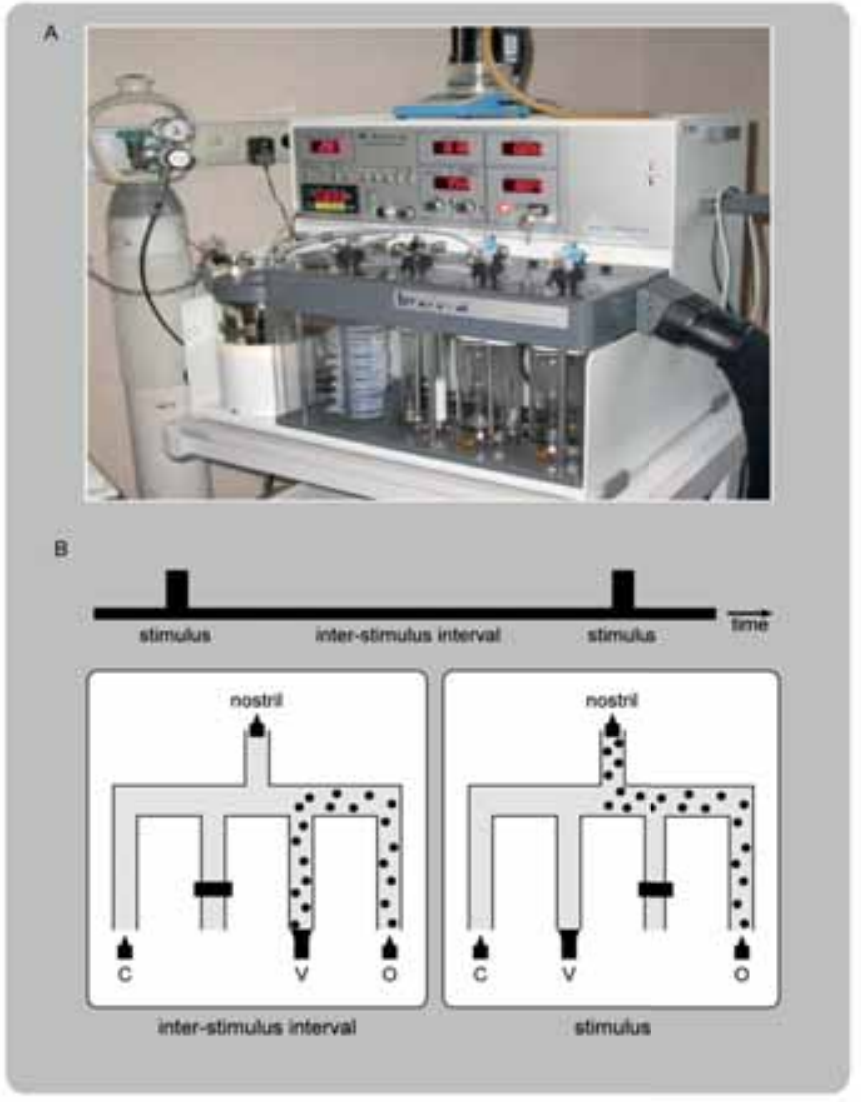

Figure 1. A). General view of the Olfactometer OM2s (Burghart Medical Technology, Wedel, Germany). B). Schematic diagram of the switching device of the constant airflow chemosensory stimulator. During the inter-stimulus interval, the subject's nose receives odourless control air $(\mathrm{C})$, whereas the odorized air $(\mathrm{O})$ is directed to the vacuum line (V). During the stimulation period the odorized air $(\mathrm{O})$ is directed to the outlet of the stimulator, whereas the control air (C) is directed toward the vacuum line (V).

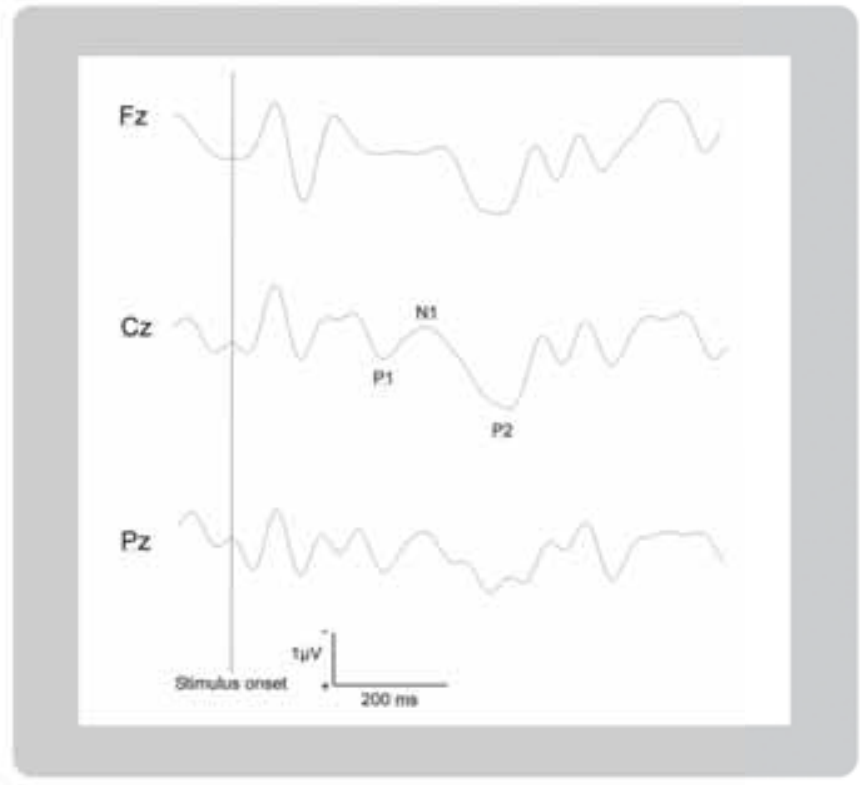

Figure 2. Olfactory chemosensory event-related potentials (ERPs) obtained using time-domain averaging in one healthy subject. 2-phenylethanol ( $50 \% \mathrm{~V} / \mathrm{V})$ was used to activate olfactory afferents selectively. 30 stimuli were presented, lasting $200 \mathrm{~ms}$ and separated by a 30-s interstimulus interval. Three distinct peaks can be identified, maximal at the scalp vertex: P1 (250 ms), N1 (416 ms) and P2 (660 ms). (Adapted from Reference (7)). rize and quantify ERS and ERD ${ }^{(23)}$. These methods rely on techniques to estimate within each EEG epoch the amplitude of the signal as a function of time and frequency, regardless of phase. The obtained time-varying expressions of oscillation amplitude are then averaged across trials, thereby disclosing both phaselocked and non-phase locked modulations of signal amplitude, provided that these modulations are relatively well time-locked to the onset of the event, and consistent in frequency. Identified in the time-frequency domain, EEG responses to sensory stimulation can be characterized by their latency, their frequency, their magnitude (often expressed as percentage relative to baseline) and their scalp distribution ${ }^{(20)}$.

To perform this time-frequency decomposition of EEG signals, several approaches have been proposed. At present, the continuous wavelet transform (CWT) is probably the most used, as it is particularly well-suited for the analysis of EEG signals. Indeed, by adapting the window width as a function of the estimated frequency, the CWT offers an optimal compromise for timefrequency resolution and is thus adequate for the evaluation of event-related modulations of the EEG spectrum within a wide range of frequencies ${ }^{(20)}$. The CWT can be performed in two ways. First, it can be applied at the level of each single EEG epochs (CWT-SINGLE). Second, it can be applied to the ERP waveforms obtained by averaging signals in the time domain (CWT-AVERAGE). The CWT-SINGLE transform enhances the signal-to-noise ratio of all time-locked EEG responses, regardless of whether they are phase-locked to the onset of the stimulus, i.e. ERPs even when subject to a significant amount of temporal jitter, ERS and ERD. In contrast, the CWT-AVERAGE average transform yields a time-frequency representation of the signals obtained using conventional time-domain averaging, and will thus contain only 


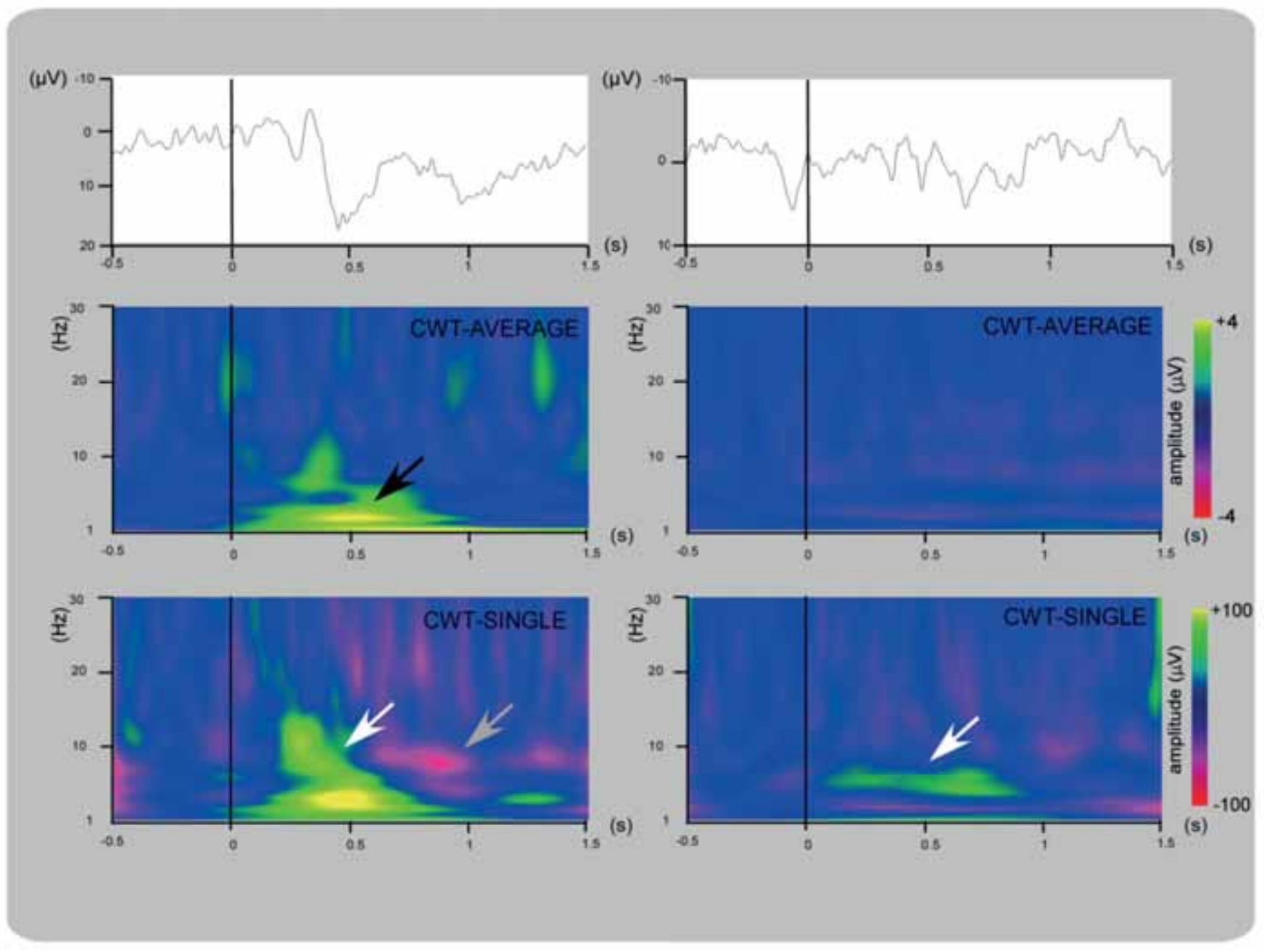

Figure 3. Phase-locked and non phase-locked event-related EEG responses to trigeminal (left panel) and olfactory (right panel) chemosensory stimulation elicited in single subjects (electrode $\mathrm{Cz}$ ). Gaseous $\mathrm{CO}_{2}(50 \% \mathrm{v} / \mathrm{v})$ was used to selectively activate trigeminal afferents. 2-Phenylethanol (50\% $/ \mathrm{v}$ ) was used to selectively activate olfactory afferents. 60 stimuli were presented, lasting $200 \mathrm{~ms}$ (rising-time 20 ms), separated by a 30 s inter-stimulus interval. The upper panels show the time-domain average ERP waveform. Following trigeminal stimulation, three distinct peaks can be identified at the scalp vertex: P1 (284 ms), N1 (342 ms) and P2 (457 ms). In contrast, no clear ERP can be identified following olfactory stimulation. The middle panels display the time-frequency representation of the phase-locked EEG responses contained in these average waveforms (CWT-AVERAGE transform). Signal increases and decreases relative to baseline $(-400--100 \mathrm{~ms}$ relative to stimulus onset) are represented in yellow and red, respectively. Note that the trigeminal chemosensory ERP (black arrow) is mainly constituted of low-frequency activity centered between 1 and $8 \mathrm{~Hz}$. Also note the lack of a clear EEG response following olfactory stimulation. The lower panels show the result of the time-frequency transform applied at the level of each single EEG epochs (CWT-SINGLE), thus revealing both phase-locked and non phase-locked stimulus-induced EEG changes. Note that in addition to the phase-locked EEG response, the trigeminal stimulus also elicits non phase-locked EEG responses (white arrows), constituting of an increase of EEG power centered around $10 \mathrm{~Hz}$, followed by desynchronization of alpha-band oscillations (grey arrow). Most importantly, note that the olfactory stimulus elicits a marked long-lasting non phase-locked increase of EEG power (white arrow).

EEG responses that are consistently phase-locked to the stimulus. Therefore, EEG responses that are visible in both the CWT-SINGLE and CWT-AVERAGE can be considered as phase-locked; whereas activities that are visible only in the CWT-SINGLE can be considered as non phase-locked (ERPs subject to jitter, ERS and ERD) (20).

Time-frequency analysis using CWT has no major limitations as compared to other EEG analyses. When estimating low frequencies the CWT uses a wide window, resulting in a low temporal resolution but high-frequency resolution. However, at these low frequencies, the loss of temporal resolution is irrelevant because the latencies of low frequency are uncertain by definition ${ }^{\left({ }^{34}\right)}$. In contrast, when estimating high frequencies, the CWT uses a narrow window, resulting in high temporal resolution but low fre- 


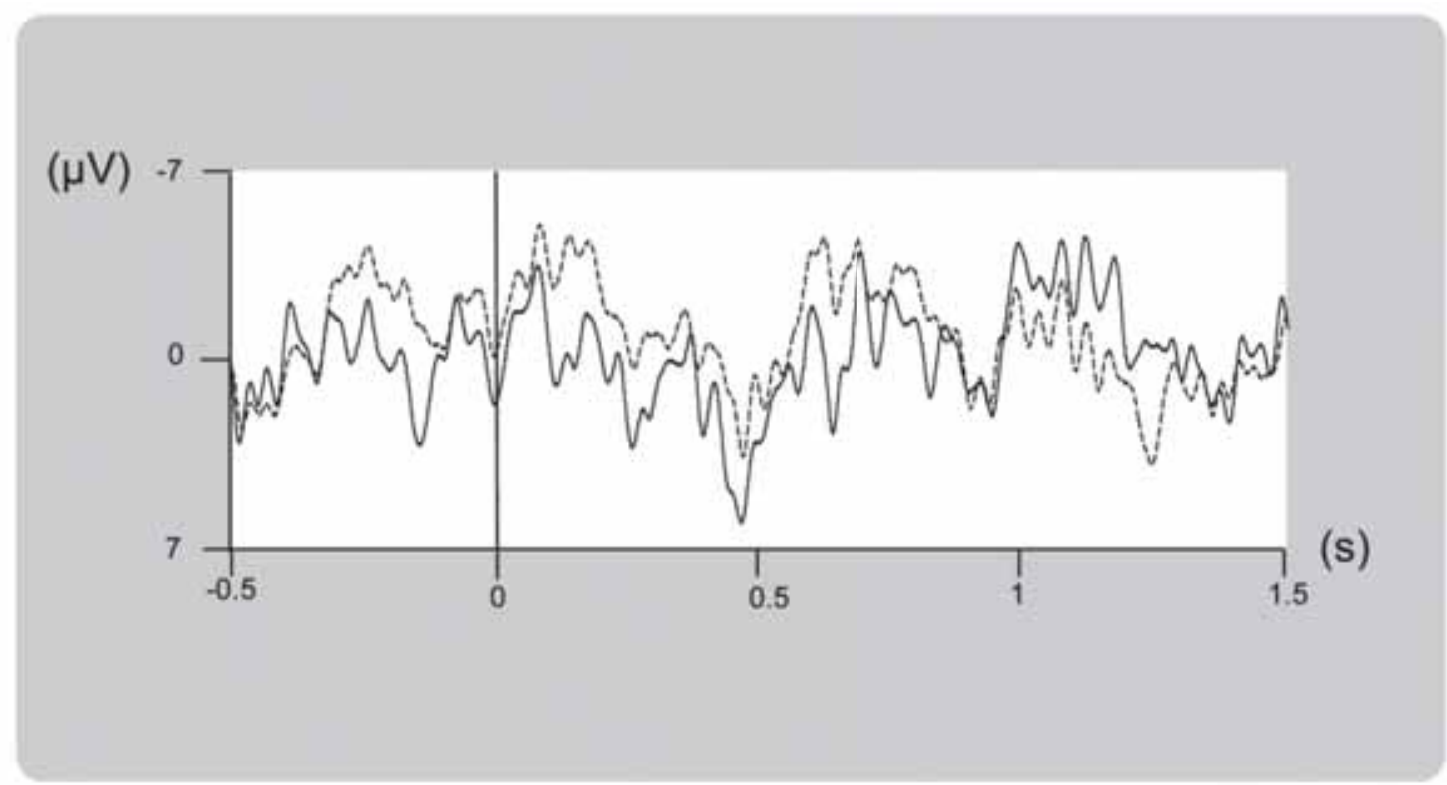

Figure 4. Increasing the signal-to-noise ratio of chemosensory ERPs using an Independent Component Analysis (ICA) to perform a blind source separation of non-cerebral electrical artifacts. The figure shows the olfactory chemosensory ERPs obtained at electrode $\mathrm{Cz}$ in one healthy subject before (dashed line) and after (continuous line) removing independent components capturing electro-ocular artifacts.

quency resolution. However, at these high frequencies, the loss of frequency resolution is irrelevant because the frequencies composing short lasting changes are irrelevant by definition.

As chemosensory stimuli may be subject to jitter and/or induced ERS or ERD, time-frequency analysis seems very interesting in the field of chemosensory event-related potentials, to reveal events that are usually lost by classical time-domain averaging.

Using local field potentials (LFP) recordings performed in animals such as rodents, some authors have already studied oscillatory neuronal activity within the olfactory bulb ${ }^{(35)}$ and olfactory cortex ${ }^{(36-38)}$, in particular, oscillatory activities in the gamma band $(>40 \mathrm{~Hz}$ ). These studies have shown that gamma oscillations occur in short bursts and possibly reflect feedback interactions between excitatory and inhibitory neurons ${ }^{(35-38)}$. They appear to be linked strongly to the inhalation cycle, being initiated at the end of the inhalation cycle and persisting into the expiratory period ${ }^{(39-41)}$. The power of odour evoked gammaband oscillations has been shown to be associated with discrimination of similar odorants ${ }^{(42,43)}$.

In humans, Jung et al., ${ }^{(44)}$ studied olfactory-induced oscillations using intracerebral electrodes implanted in the amygdale for therapeutic purposes. They performed a time-frequency analysis of the recorded signals and showed that odorants induce oscillatory responses in this structure, both in the beta-band (15 - 25 $\mathrm{Hz}$ ) and in the gamma-band ( $25-35 \mathrm{~Hz})$, hypothesized to play a role in the coding of olfactory information. Using non-invasive surface EEG, Masaoka et al., ${ }^{(45)}$ reported inspiratory phase-locked alpha-band oscillations after the occurrence of an olfactory stimulus. However, these studies examined the frequency contents of the signals after time-domain averaging, which is likely to have largely canceled out non phase-locked ERS and ERD. Lorig and Randol (46) explored ERS and ERD following stimulation with different odorants. They didn't use the CWT but the method described by Pfurtscheller ${ }^{(47)}$. They showed that most electrophysiological differences between different odorants occurred $1.5-2.0 \mathrm{~s}$ following stimulation. Differences were found in the $12-15,20-23$ and $24-27 \mathrm{~Hz}$ bands. They also noted that low frequency activity $(0.5-3 \mathrm{~Hz})$ was found to differ between chemosensory stimuli from $0.5-1.0$ s following stimulation.

As illustrated in the following example, characterizing non phase-locked EEG responses to trigeminal and olfactory chemosensory stimulation using methods such as the continuous wavelet transform could prove very useful (Figure 3). In this example, time-frequency analysis was applied to both trigeminal and olfactory responses recorded using scalp EEG in a single subject. Following trigeminal stimulation, the CWT-AVERAGE transform (which enhances only phase-locked EEG responses) shows a signal increase corresponding to the ERP. The CWTSINGLE transform (which enhances both phase-locked and non phase-locked EEG responses) reveals that in addition to the ERP, the trigeminal stimulus also elicits a non phase-locked increase (ERS) in EEG power at higher frequencies ( 9 - $15 \mathrm{~Hz}$ ), followed by 
a long lasting decrease of EEG power centered within the alphaband (ERD). As often observed, following olfactory stimulation, the CWT-AVERAGE transform does not reveal any consistent phase-locked EEG response. In contrast, the CWT-SINGLE transform reveals a consistent increase in EEG power within the theta band ( $4-8 \mathrm{~Hz}$ ), which could correspond to an ERP subject to a significant amount of temporal jitter. Furthermore, this increase of EEG power is followed by ERD occurring in the alpha-band.

Time-frequency analysis of EEG responses elicited by chemosensory stimuli thus gives additional information in comparison to time-domain averaging by disclosing an important fraction of the cortical elicited activity that is usually lost by conventional time-domain averaging. This analysis allows the identification of an ERP even if affected by a significant amount of jitter. It also allows the identification, characterization and quantification of ERS and ERD, which reflect an increase or decrease in the synchrony of underlying neuronal population. Since ERS and ERD reflect the processing of the sensory information, they may appear useful in the future to better understand neuronal processing of chemosensory information. Since this analysis provides information that is usually lost by time-domain averaging; it could increase the sensitivity and specificity of CSERP. Further studies should thus be realized to investigate the potential use of this technique in the clinical assessment of patients having an olfactory disorder.

\section{Spatio-temporal filtering of chemosensory ERPs using independent component analysis}

The EEG signal recorded on the scalp is often contaminated by non-cerebral electrical activity such as artifacts due to eye movements or heartbeat. Hence, the EEG may be viewed as the sum of several temporally overlapping and spatially distinct sources of cerebral and non-cerebral activities. Blind source separation algorithms are used in signal processing to recover independent sources from signals obtained from sensors that record a linear mixture of these sources. Therefore, these algorithms can be used to separate cerebral and non-cerebral activities contributing to the EEG, provided that these have a fixed scalp distribution. Independent component analysis (ICA) has been used successfully by a growing number of investigators to unmix EEG signals into a single linear combination of independent components (ICs), each having a maximally independent time-course and a fixed scalp distribution. Applied to non-averaged multi-channel EEG recordings, the method has proven very efficient in isolating and removing non-neural artifacts such as artifacts related to eye blinks ${ }^{(48,49)}$. More recently, the technique has also been used to separate spatially- and temporally-distinct components of ERPs ${ }^{(50,51)}$.

As shown in Figure 4 blind source separation using ICA could be used to remove non-cerebral electrical artifacts contaminating chemosensory ERPs and, thereby, increase the signal-to-noise ration of the elicited responses.

\section{Event-related source imaging}

It is generally agreed that EEG has a high temporal resolution but a poor spatial resolution, whereas techniques based on hemodynamic measures (e.g. BOLD fMRI) have a poor temporal resolution but a high spatial resolution ${ }^{(21)}$. Although source analysis techniques are more appropriate to localize signals originating close to the scalp surface, several recent studies have suggested that EEG responses originating from deep brain structures can also be recorded and localized accurately ${ }^{(52,53)}$. Source localization methods rely on mathematical models of the bio-electrical generators and the volume conductors within which they lie. The key limitation of these methods is that the inverse problem is highly underdetermined and some assumptions have to be made when solving it. Hence, the validity of the obtained source configurations is strongly conditioned by the validity of these assumptions (e.g. assumptions concerning the number of sources or their approximate location, etc.). Source analysis of CSERP is probably particularly problematic, not only because of the relatively deep location of the hypothesized sources, but also because multiple bilateral sources are thought to be simultaneously active, thus making it extremely difficult to draw significant conclusions. Nevertheless, using high-resolution EEG, some researchers have attempted to localize the cortical structures generating the different components of CSERPs ${ }^{54,}$ 55). For example, in an attempt to provide information on the spatio-temporal sequence of information processing in the olfactory pathway, Lascano et al., ${ }^{(56)}$ performed of source analysis of olfactory CSERPs, in which he suggested that olfactory input is processed first in the mesial and lateral temporal cortex of the hemisphere ipsilateral to the stimulated nostril, and only subsequently in the corresponding structures of the contralateral hemisphere.

\section{Conclusion}

The recording of olfactory CSERPs constitutes a promising approach to explore the cortical processing of odours in humans. However, at present, the low signal-to-noise ratio of the obtained responses has constituted an important limitation. This low signal-to-noise ratio could be due, at least in part, to the fact that conventional time-domain averaging cancels out a significant fraction of the stimulus-evoked cortical activity, in particular, ERPs affected by temporal jitter, ERD and ERS. Recent signal-processing methods have been introduced (1) to reveal stimulus-induced EEG responses that are not phaselocked to the stimulus, and (2) to increase the signal-to-noise ratio of the elicited responses using spatio-temporal filtering. Altogether, these new techniques could help to characterize better the cortical processing of odours in humans. 


\section{References}

1. Moncrief RW. Effect of odours on EEG records. Perfumery essential oil Rec. 1962 53: 757-760.

2. Alisson T, Goff WR. Human cerebral evoked responses to odorous stimuli. Electroencephalogr Clin Neurophysiol. 1967; 23: 558-560.

3. Aufferman H, Gerull G, Mathe F, Mrowinski D. Olfactory evoked potentials and negative variation simultaneously recorded for diagnosis of smell disorder. Ann Otol Rhinol Laryngol. 1993; 102: 6-10.

4. Finkenzeller P. Gemittelte EEG-Pontentiale bei olfactorischer ReizungPotentiale bei olfaktorischer Reizung. Pflügers Arch. 1966; 292: 76-80.

5. Kobal G, Plattig KH. Metodische Anmerkungen zur Gewinnung olfaktorischer EEG-Antworten des wachen Menschen (objektive Olfaktometrie). Z EEG-MEG. 1978; 9: 135-145

6. Laudien JH, Wencker S, Ferstl R, Pause BM. Context effects on odor processing: an event-related potential study. Neuroimage 2008; 41: 1426-1436.

7. Rombaux P, Mouraux A, Bertrand B, Guerit $J M$, Hummel T. Assessment of olfactory function using chemosensory event-related potentials. Neurophysiol Clin. 2006; 36: 53-62.

8. Pause BM, Krauel J. Chemosensory eventrelated potentials (CSERP) as a key to psychology of odors. Int J Psychophysiol. 2000; 36: 105-122.

9. Welge-Lüssen A, Wille C, Renner B, Koba G. Test-retest reliability of chemosensory evoked-potentials. J Clin Neurophysiol. 2003; 20: 135-142.

10. Thesen T, Murphy C. Reliability analysis of event-related brain potentials to olfactory stimuli. Psychophysiology. 2002; 39: 733-738

11. Boesveldt $S$, Haehner A, Berendse $H$ Hummel T. Signal-to-noise ratio of chemosensory event-related potentials. Clin Neurophysiol. 2007; 118: 690-695

12. Lötsch J, Hummel T. The clinical significance of electrophysiological measures of olfactory function. Behav Brain Res. 2006; 170: 78-83.

13. Rombaux P, Bertrand B, Keller T, Mouraux A. Clinical significance of olfactory eventrelated potentials related to orthonasal and retronasal olfatcory testing. Laryngoscope. 2007; 117: 1093-1101

14. Kassab A, Schaub F, Vent J, Hüttenbrink KB, Damm M. Effects of short interstimulus intervals on olfactory and trigeminal eventrelated potentials. Acta Otolaryngol. 2009; 129: $1250-1256$

15. Hummel T, Kobal G. Chemosensory eventrelated potentials to trigeminal stimuli change in relation to the interval between repetitive stimulation of the nasal mucosa. Eur Arch Otolaryngol. 1999; 256: 16-21.

16. Hummel T, Kobal G, Gudziol H, Mackay$\operatorname{Sim}$ A. Normative data for the Sniffin Sticks including test of odor identification, odor discrimination and or odor thresholds: an upgrade based on a group of more than 3000 subjects. Eur Arch Otorhinolaryngol. 2007; 264: 237-243
17. Kobal G, Hummel T, Sekinger B, Barz S, Roscher S, Wolf S. "Sniffin' Sticks": screening of olfactory performance. Rhinology. 1996; 34: 222-226.

18. Doty RL, Shaman P, Kimmelman CP, Dann MS. University of Pennsylvania Smell Identification Test: a rapid quantitative olfactory function test for the clinic. Laryngoscope. 1984; 94: 176-178.

19. Mouraux A, Guerit JM, Plaghki L. Nonphase locked electroencephalogram (EEG) responses to $\mathrm{CO} 2$ laser skin stimulation may reflect central interactions between A partial differential- and C-fibre afferent volleys. Clin Neurophysiol. 2003; 114: 710-722.

20. Mouraux A, lannetti GD. Across-trial averaging of event-related EEG responses and beyond. Magn Reson Imaging. 2008; 1041 1054.

21. Luck SJ. An introduction to the event-related potential technique. Cambrige: MIT Press; 2005; p30-31.

22. Berger H. Über das elektroenkephalogramm des menschen. Arch Psychiatry. 1929; 8: 527570.

23. Pfurtscheller G, Lopes da Silva FH. Eventrelated EEG/MEG synchronization and desynchronization: basic principles. Clin Neurophysiol. 1999; 110: 1842-1857.

24. Singez W. Synchronization of cortical activity and its putative role in information processing and learning. Annu Rev Physiol. 1993: 55: 349-374.

25. Schulz E, Tiemann L, Schuster T, Gross J, Ploner M. Neurophysiological coding of traits and states in the perception of pain. Cereb Cortex. 2011 doi: 10.1093/cercor/bhr027.

26. Cui L, Evans WJ. Olfactory event-related potentials to amyl acetate in congenital anosmia. Electroencephalogr Clin Neurophysiol. 1997; 102: 303-306.

27. Evans WJ, Cui L, Starr A. Olfactory eventrelated potentials in human subjects; effects of age and gender. Electroencephalogr Clin Neurophysiol. 1995; 95: 293-301.

28. Hummel T, Barz S, Pauli E, Kobal G. Chemosensory event-related potentials change with age. Electroencephalogr Clin Neurophysiol. 1998; 108: 208-217.

29. Hummel T, Kobal G. Olfactory event-related potentials. In: Simon SA, editor. Methods and New frontiers in Neurosciences. Boca Raton, FL: CRC Press; 2002.

30. Pause BM, Sojka B, Krauel K, Fehn-Wolfsdorf $G$, Ferst R. Olfactory information processing during the course of the menstrual cycle. Biol Psychol. 1996; 44: 31-54.

31. Morgan CD, Covington JW, Geisler MW Polich J, MurphyC. Olfactory event-related potentials; older males demonstrate the greatest deficits. Electroencephalogr Clin Neurophysiol. 1997; 104: 351-358.

32. Morgan CD, Geisler MW, Covington JW, Polich J, Murphy C. Olfactory P3 in young and older adults. Psychophysiol. 1999; 36: 281-287.

33. Pause B, Krauel K, Sojka B, Fesrtl R. Is odour processing related to oral breathing? Int J Psychophysiol. 1999: 32: 251-260.

34. Baker SN, Curio G, Lemon RN. EEG oscillations at $600 \mathrm{~Hz}$ are macroscopic markers for cortical spike bursts. J Physiol. 2003; 550(Pt2): 529-534.

35. Adrian ED. Olfactory reactions in the brain of the hedgehog. J Physiol. 1942; 100: 459-473.

36. Boudreau J-C, Freeman W. Spectral analysis of electrical activity in the prepyriform cortex of the cat. Exp Neurol. 1963: 8: 423-430.

37. Freeman WJ. Distribution in time and space of prepyriform electrical activity. J Neurophysiol. 1959; 22: 644-665.

38. Freeman WJ. Correlation of electrical activity of prepyriform cortex and behavior in cat. J. Neurophysiol. 1960; 23: 111-131.

39. Rojas-Libano D, Kay LM. Olfactory system gamma oscillations: the physiological dissection of cognitive neural system. Cogn Neurodyn. 2008; 2: 179-194.

40. Kay LM, Beshel J, Brea J, Martin C, RojasLibano D, Kopell N. Olfactory oscillations: the what, how and what for. Trends Neurosci. 2009: 32: 207-214.

41. Buonviso N, Amat C, Litaudon P. Respiratory modulation of olfactory neurons in the rodent brain. Chem Senses. 2006; 31: 145154

42. Beshel J, Kopell N, Kay LM. Olfactory bulb gamma oscillations are enhanced with task demands. J Neurosci. 2007; 27: 8358-8365.

43. Stopfer M, Bhagavan S, Smith BH, Laurent G. Impaired odour discrimination on desynchronization of odour-encoding neural assemblies. Nature. 1997; 390: 70-74.

44. Jung J, Hudry J, Ryvlin P, Royet JP, Bertrand $O$, Lachaux JP. Functional significance of olfactory-induces oscillations in the human amygdale. Cereb Cortex. 2006; 16: 1-8.

45. Masaoka Y, Koiwa N, Homma I. Inspiratory phase-locked alpha oscillation in human olfaction: source generators estimated by a dipole tracing method. J Physiol. 2005; 566: 979-997.

46. Lorig TS, Randol M. Evaluation of eventrelated synchronous brain activity following chemosensory stimulation. Chem Senses. 1996; 21: 636.

47. Pfurtscheller G. Event-related synchronization ERS: an electrophysiological correlate of areas at rest. Electroencephalogr Clin Neurophysiol. 1992; 83: 62-69.

48. Jung TP, Makeig S, Westerfield M, Townsend J, Courchesne E, Sejnowski TJ. Removal of eye activity artifacts from visual event-related potentials in normal and clinical subjects. Clin Neurophysiol. 2000; 111: 1745-1758.

49. Makeig S, Enghoff S, Jung TP, Sejnowski TJ. Moving-window ICA decomposition of EEG data reveals event-related changes in oscillatory brain activity. 2nd Int. Workshop on Independent Component Analysis and Signal Separation; 2000; P.627-632.

50. Makeig S, Jung TP, Bell AJ, Ghahremani D, Sejnowski TJ. Blind separation of auditory event-related brain responses into independent components. Proc Natl Acad Sci U S A. 1997; 94: 10979-10984.

51. Makeig S, Debener S, Onton J, Delorme A. Mining event-related brain dynamics. Trends Cogn Sci. 2004; 8: 204: 10

52. Attal Y, Bhattacharjee M, Yelnik J, et al Modeling and detecting deep brain activity 
with MEG and EEG. Conf Proc IEEE Eng Med Biol Soc. 2007: 4937-4940.

53. Zumsteg D, Friedman A, Wennberg RA Wieser HG. Source localization of mesial temporal interictal epileptiform discharges: correlation with intracranial foramen ovale electrode recordings. Clin Neurophysiol. 2005; 16: 2810-2818.

54. Kettenmann B, Hummel T, Kobal G. Functional imaging of olfactory activation in the human brain. In: Methods and frontiers in chemosensory research. Simon SA, Nicolelis MAL, eds, pp477-506. Baco Raton, FL: CRC press.
55. Miyanari A, Kaneoke $Y$, Ihara $A$, et al. Neuromagnetic changes of brain rhythm evokes by intravenous olfactory stimulation in humans. Brain Topogr. 2006; 18: 189-199.

56. Lascano AM, Hummel T, Lacroix JS, Landis BN, Michel CM. Spatio-temporal dynamics of olfactory processing in the human brain: an event-related source imaging study. Neuroscience. 2010; 167: 700-708.
Prof Philippe Rombaux Department of Otorhinolaryngology Cliniques Universitaires Saint-Luc Avenue Hippocrate 10 1200 Brussels Belgium

Tel: +32-2-764 1949

E-mail: philippe.rombaux@uclouvain.be

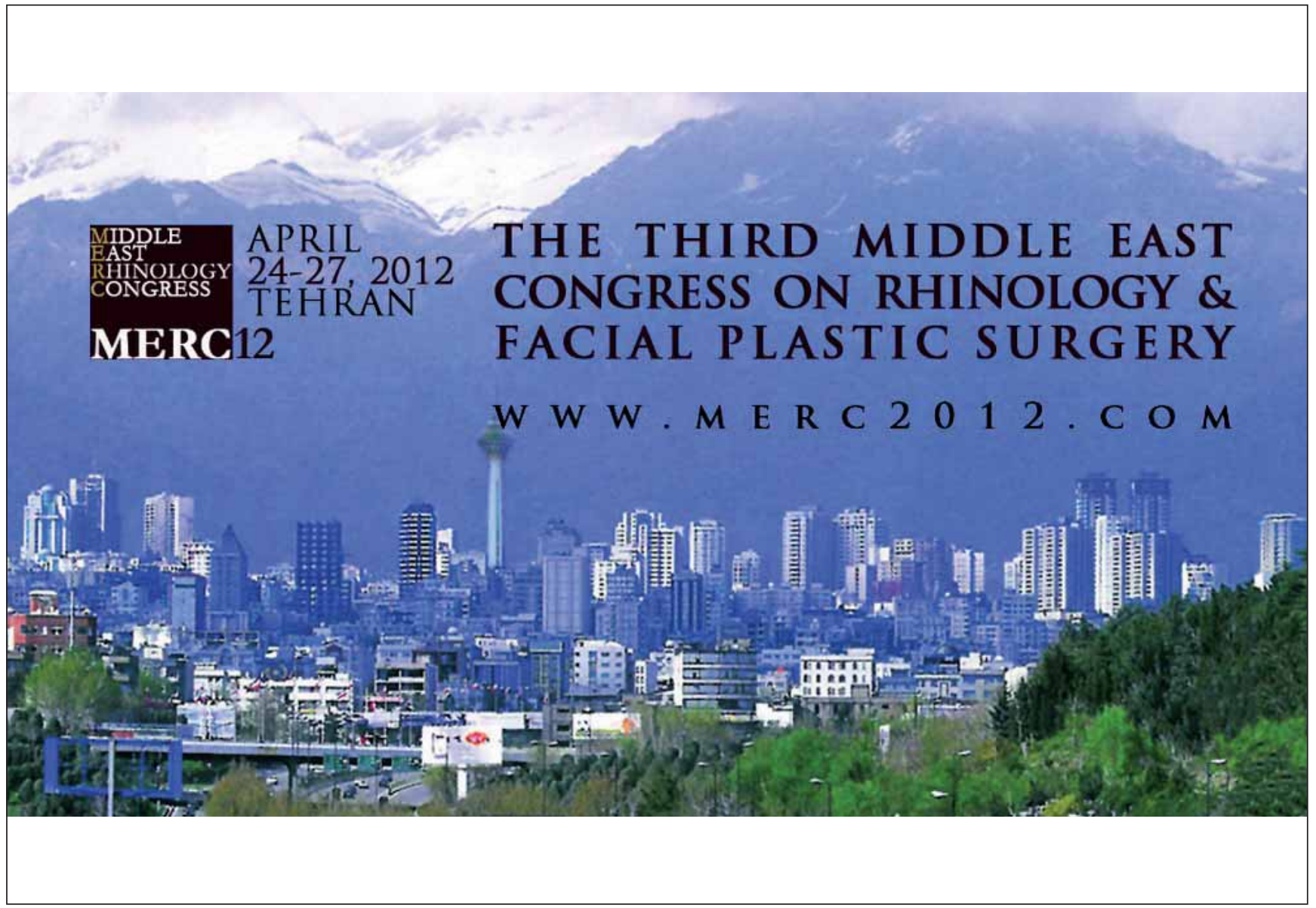

